\title{
Fotoproteção em Agentes Comunitários de Saúde (ACS) de Belém-PA
}

\section{Photoprotection of Community Health Workers (CHW) of Belém-PA \\ Fotoprotección en Agentes Comunitarios de Salud (ACS) de Belém-PA}

\author{
Claudia Marques Santa Rosa Malcher ${ }^{1,2} \oplus$, Alan Luz Tembra ${ }^{2} \oplus$, Felipe Costa Amorim²®, Thalles Ricardo Melo de Souza ${ }^{2} \oplus$, \\ Monaliza dos Santos Pessoa² ${ }^{2}$ \\ ${ }^{1}$ Programa de Pós-Graduação em Oncologia e Ciências Médicas (PPGOCM) pela Universidade Federal do Pará (UFPA). Belém, PA, Brasil. \\ 2 Universidade do Estado do Pará (UEPA). Belém, PA, Brasil.
}

\section{Resumo}

Objetivo: Avaliar a fotoproteção dos Agentes Comunitários de Saúde (ACS) de 5 equipes da Estratégia Saúde da Família. Métodos: A casuística consistiu em 56 ACS, os quais foram submetidos a um questionário autoaplicável com perguntas sobre os hábitos de exposição solar e os conhecimentos acerca do assunto. Resultados: Verificou-se que $48,2 \%$ dos ACS se expunham ao sol durante 2 a 6 horas entre segunda e sexta-feira e que 55,4\% deles tinham exposição entre 10 e 15 horas. Além disso, 58,9\% dos ACS utilizam filtros solares raramente ou nunca e, entre esses, $53,6 \%$ os aplicam com frequência inadequada. Contudo, $100 \%$ dos pesquisados relataram conhecer as consequências negativas da exposição solar. Conclusão: A fotoproteção dos ACS foi considerada inadequada, já que poucos utilizam filtros solares e os que relataram o uso do produto o faziam de maneira inapropriada. Ademais, o conhecimento sobre os riscos e as consequências da exposição solar foi considerado excelente.

Palavras-chave: Protetores Solares; Agentes Comunitários de Saúde; Prevenção Primária; Atenção Primária à Saúde; Saúde do Trabalhador

\begin{abstract}
Objective: To evaluate the photoprotection of Community Health Agents $(\mathrm{CHA})$ in five health units. Methods: A casuistry of $56 \mathrm{CHA}$ was used, which were submitted to a self-administered questionnaire with questions about their solar exposure habits and their knowledge about this subject matter Results: From the analysis of the obtained data, it was verified that $48.2 \% \mathrm{CHA}$ exposed themselves to the sun during 2 to 6 hours between Monday and Friday and that $55.4 \%$ of them are exposed between $10 \mathrm{AM}$ and $3 \mathrm{PM}$. Furthermore, $58.9 \%$ of $\mathrm{CHA}$ rarely or never use solar filters and among them, $53.6 \%$ apply the product with an inappropriate frequency. However, $100 \%$ of the CHA reported to know about the negative consequences and the risks of the solar exposure. Conclusion: The agents' photoprotection was considered inappropriate, since just a few of them use solar filters and the ones, which reported using the product, do it improperly. Besides that, their knowledge about the risks and consequences of the solar exposure was considered excellent.
\end{abstract}

Keywords: Sunscreening Agents; Community Health Workers; Primary Prevention; Primary Health Care; Occupational Health

Como citar: Malcher CMSR, Tembra AL, Amorim FC, Souza TRM, Pessoa MS. Fotoproteção em Agentes Comunitários de Saúde (ACS) de Belém-PA. Rev Bras Med Fam Comunidade. 2019;14(41):1798. http://dx.doi.org/10.5712/rbmfc14(41)1798
Autor correspondente: Claudia Marques Santa Rosa Malcher. E-mail: claudiaufpa@gmail.com Fonte de financiamento: declaram não haver. Parecer CEP: 2.451.059 (UEPA), aprovado em 20/12/2017.

Procedência e revisão por pares: revisado por pares. Recebido em: 25/07/2018. Aprovado em: 19/11/2018. 


\section{Resumen}

Objetivo: Evaluar la fotoprotección de los Agentes Comunitarios de Salud (ACS) de cinco unidades estudiadas. Métodos: La casuística consistió en 56 ACS, los cuales han sido sometidos a un cuestionario autoaplicable con preguntas sobre los hábitos de exposición solar y los conocimientos sobre el asunto. Resultados: Se verificó que el $48,2 \%$ de los ACS se exponían al sol durante 2 a 6 horas entre el lunes y el viernes y que el $55,4 \%$ de ellos se exponen entre las 10 de la mañana y 3 de la tarde. Además, el 58,9\% de los ACS utilizan filtros solares raramente o nunca, y, entre ellos, $53,6 \%$ los aplican con frecuencia inadecuada. Sin embargo, el 100\% de los entrevistados relataron conocer las consecuencias negativas de la exposición al sol. Conclusión: La fotoprotección de los ACS fue considerada inadecuada, en razón de la poca utilización de filtros solares y los que relataron el uso del producto lo hacían de manera inadecuada. Además, el conocimiento sobre los riesgos y las consecuencias de la exposición solar fue considerado excelente.

Palabras clave: Protectores Solares; Agentes Comunitarios de Salud; Prevención Primaria; Atención Primaria de Salud; Salud Laboral

\section{Introdução}

O Programa Saúde da Família (PSF), atual Estratégia Saúde da Família (ESF) desde 2006 é a base do Programa Nacional de Atenção Básica (PNAB), implantado no Brasil em 1994 com objetivo de centralizar a saúde no indivíduo, compreendendo-o de maneira mais integral e assumindo o compromisso de desfazer o modelo médico-hegemônico até então vigente. Dessa forma, a ESF busca consolidar os princípios e as diretrizes do Sistema Único de Saúde (SUS) por meio de trabalhadores, que desenvolvem ações de prevenção, promoção e proteção da saúde. ${ }^{1}$

A equipe da ESF (eSF) é multiprofissional e composta por, no mínimo, um médico, generalista ou de família, um enfermeiro, um técnico de enfermagem e pelos Agentes Comunitários de Saúde (ACS). ${ }^{2}$ Nesse cenário, é válido destacar o papel dos ACS, os quais atuam como mediadores e como um elo que aproxima a comunidade local à ESF, o que facilita a criação de vínculos de confiança entre a mesma e a comunidade. Sendo assim, os ACS figuram como forte apoio para reestruturar a atenção básica. ${ }^{3}$

Grande parte das atividades dos ACS é feita fora do âmbito da unidade de saúde que abriga a equipe de Saúde da Família, estando eles sujeitos às intempéries do ambiente, como o sol e a chuva. ${ }^{4}$ Assim, é válido voltar a nossa atenção para a exposição ao sol por parte dos ACS, pois sabe-se que a radiação ultravioleta solar, apesar de trazer benefícios para a saúde humana, traz também diversos riscos, como o câncer de pele, o envelhecimento precoce e variadas lesões cutâneas. ${ }^{5}$

É consenso na literatura médica que a radiação solar está diretamente relacionada ao câncer de pele, tanto os melanomas quanto os não melanomas (carcinoma basocelular e espinocelular). ${ }^{6}$

Dessa forma, uma das melhores maneiras de realizar a prevenção contra o câncer de pele e outras complicações relacionadas à exposição solar é por meio da fotoproteção, que pode ser feita de diferentes formas, sendo as principais: fotoprotetores tópicos (filtros solares químicos ou físicos) e a fotoproteção mecânica (roupas, chapéus, óculos de sol, sombras e vidros), além da fotoproteção oral (nutricosméticos ou nutricêuticos). ${ }^{7}$

Diante do exposto, visto a importância dos ACS na atenção básica e a intensa exposição solar a que estão sujeitos no exercer de suas funções, o presente estudo objetivou avaliar a fotoproteção dos ACS das unidades estudadas. 


\section{Método}

\section{Desenho do Estudo}

O presente estudo é de natureza quantitativa, de cunho transversal e descritivo, feito com base nas respostas dos questionários aplicados.

\section{Cenário do estudo}

Cinco eSF de dois bairros do município de Belém, no Pará, foram escolhidas devido à parceria com a Universidade do Estado do Pará (UEPA), o que facilitava a comunicação entre as equipes das unidades e os pesquisadores. Atualmente, neste município encontram-se registradas junto ao Ministério da Saúde um total de 53 eSF.

\section{Participantes e Critérios de Inclusão e Exclusão}

A população do estudo foi de 64 ACS pertencentes às eSF selecionadas, dos quais houve perdas e foram excluídos 4 ACS por estarem de férias no período da coleta e 4 que faltaram no momento marcado com os pesquisadores.

\section{Metodologia Proposta}

Consistiu em um questionário autoaplicável, com perguntas sobre os respectivos hábitos de exposição solar e os conhecimentos acerca do assunto de cada entrevistado, segundo protocolo elaborado pelos pesquisadores, adotando-se como parâmetro principal de avaliação o atual Consenso Brasileiro de Fotoproteção. $^{7}$

Os questionários foram aplicados no âmbito das eSF, em momento previamente marcado com os ACS, e supervisionado pelos pesquisadores a fim de diminuir vieses no estudo. Foi considerada a prevalência de $50 \%$ para o desfecho dessa investigação.

\section{Análise de dados}

Os dados foram registrados e organizados no pacote Microsoft Office 2010 fornecendo informações necessárias para formulações e tabelas. A análise estatística dos dados foi feita com intervalo de confiança de proporção com a significância de 95\%.

\section{Aspectos Éticos}

A pesquisa obedeceu às normas contidas na resolução 466/12 do Conselho Nacional de Saúde que dispõe sobre as pesquisas envolvendo seres humanos. Os participantes assinaram o Termo de Consentimento Livre e Esclarecido e foram informados dos objetivos da pesquisa e comunicados que poderiam retirar seu nome em qualquer momento. O projeto foi submetido ao Comitê de Ética em Pesquisa da UEPA e aprovado sob o parecer de $n^{\circ} 2.451 .059$. 


\section{Resultados}

O perfil dos ACS entrevistados está detalhado na Tabela 1. Os participantes foram predominantemente do sexo feminino, a maioria tem pelo menos o ensino médio completo e todos trabalhavam há mais de um ano como ACS.

Tabela 1. Perfil dos Agentes Comunitários de Saúde de Belém-PA, em 2018.

\begin{tabular}{|c|c|c|}
\hline Caracterização & $\mathbf{N}$ & $\%$ \\
\hline \multicolumn{3}{|l|}{ Faixa Etária } \\
\hline $26-31$ & 13 & 23,6 \\
\hline $32-37$ & 11 & 20,0 \\
\hline $38-43$ & 12 & 21,8 \\
\hline $44-49$ & 14 & 25,5 \\
\hline $50-55$ & 4 & 7,3 \\
\hline $56-61$ & 1 & 1,8 \\
\hline \multicolumn{3}{|l|}{ Sexo } \\
\hline Masculino & 14 & 25,5 \\
\hline Feminino & 41 & 74,5 \\
\hline \multicolumn{3}{|l|}{ Grau de Escolaridade } \\
\hline Superior completo & 11 & 19,6 \\
\hline Superior incompleto & 17 & 30,4 \\
\hline Médio completo & 25 & 44,6 \\
\hline Médio incompleto & 3 & 5,4 \\
\hline \multicolumn{3}{|c|}{ Tempo de Serviço como ACS ${ }^{* * *}$} \\
\hline Mais de 1 ano & 56 & 100 \\
\hline \multicolumn{3}{|c|}{ Fototipo na escala de Fitzpatrick } \\
\hline I-Branca & 7 & 12,5 \\
\hline II - Branca & 8 & 14,3 \\
\hline III - Morena Clara & 15 & 26,8 \\
\hline IV - Morena Moderada & 15 & 26,8 \\
\hline V - Morena Escura & 8 & 14,3 \\
\hline VI-Negra & 3 & 5,4 \\
\hline
\end{tabular}

*** Agentes Comunitários de Saúde. Nota: 1 ACS não preencheu os dados sexo e idade, computando somente 55 ACS para a análise dessas variáveis.

Os hábitos de exposição solar dos ACS estão detalhados na Tabela 2.

As principais medidas de fotoproteção adotadas pelos ACS são detalhadas na Tabela 3. Cabe destacar que as variáveis "FPS utilizado e/ou considerado mais adequado pelos ACS" e "principal fonte de orientação para escolha do protetor" foram opcionais e marcadas de acordo com o conhecimento de cada ACS e que a variável "itens de proteção mecânica" admitiu mais de uma resposta.

Quanto ao conhecimento acerca dos riscos e consequências negativas da exposição solar, $100 \%$ dos ACS relataram conhecer algum, sendo os mais comuns relatados na Tabela 4. 
Tabela 2. Hábitos de exposição solar dos Agentes Comunitários de Saúde de Belém-PA, em 2018.

\begin{tabular}{lccc}
\hline Caracterização & $\mathbf{n}$ & $\%$ & IC95\% \\
\hline Tempo de exposição solar de segunda à sexta & & & \\
\hline Até 2 horas & 9 & 16,1 & $(6,4-25,7)$ \\
De 2 a 6 horas & 27 & 48,2 & $(35,1-61,2)$ \\
Mais de 6 horas & 20 & 35,7 & $(23,1-48,2)$ \\
\hline Horário de exposição ao sol & & & \\
\hline Até as 10 horas da manhã & 23 & 31,1 & $(18,9-43,2)$ \\
Entre 10 horas da manhã e 3 horas da tarde & 41 & 55,4 & $(42,3-68,4)$ \\
Após as 3 horas da tarde & 7 & 9,5 & $(1,8-17,1)$ \\
Não me exponho ao sol & 3 & 4,1 & $(0-9,2)$ \\
\hline
\end{tabular}

IC95\%: Intervalo de confiança 95\%. Nota: a variável "horário de exposição ao sol" admitiu mais de uma resposta.

Tabela 3. Medidas de fotoproteção adotadas pelos Agentes Comunitários de Saúde de Belém-PA, em 2018.

\begin{tabular}{|c|c|c|c|}
\hline Caracterização & $\mathbf{N}$ & $\%$ & IC95\% \\
\hline Diariamente & 13 & 23,2 & $(12,1-34,2)$ \\
\hline $\begin{array}{l}\text { Eventualmente, em qualquer estação, quando se expõe a sol } \\
\text { muito intenso }\end{array}$ & 3 & 5,4 & $(0-11,3)$ \\
\hline Nunca & 12 & 21,4 & $(10,6-32,1)$ \\
\hline \multicolumn{4}{|l|}{ FPS $^{\star \star \star}$ utilizado e/ou considerado mais adequado pelos ACS } \\
\hline Até 15 & 1 & 2,3 & $(0-6,2)$ \\
\hline Entre 15 e 30 & 15 & 33,3 & $(20,9-45,6)$ \\
\hline Farmacêutico & 2 & 3,6 & $(0-8,4)$ \\
\hline Mídia & 4 & 7,1 & $(0-13,8)$ \\
\hline Autoindicação & 20 & 35,7 & $(23,1-48,2)$ \\
\hline Outros & 2 & 3,6 & $(0-8,4)$ \\
\hline Não soube informar & 7 & 12,5 & $(3,8-21,1)$ \\
\hline \multicolumn{4}{|l|}{ Frequência de aplicação diária do filtro solar } \\
\hline Nenhuma vez & 16 & 28,6 & $(16,7-40,4)$ \\
\hline Uma vez & 21 & 37,5 & $(24,8-50,1)$ \\
\hline Duas vezes & 8 & 14,3 & $(5,1-23,4)$ \\
\hline Não utilizo nenhum dos itens acima & 9 & 12,2 & $(3,6-20,7)$ \\
\hline
\end{tabular}


Tabela 4. Conhecimentos acerca dos riscos da exposição solar dos Agentes Comunitários de Saúde de Belém-PA, em 2018.

\begin{tabular}{lccc}
\hline Caracterização & $\mathbf{n}$ & $\%$ & IC95\% \\
\hline Danos e consequência da exposição solar & & & \\
\hline Conhece & 56 & 100 & $1-1$ \\
$\quad$ Desconhece & 0 & 0 & $0-0$ \\
\hline Riscos da exposição solar & & & \\
\hline Câncer de pele & 52 & 26,7 & $(15,1-38,2)$ \\
Queimaduras solares & 41 & 21,0 & $(10,3-31,6)$ \\
Manchas & 51 & 26,1 & $(14,5-37,6)$ \\
Envelhecimento da pele & 46 & 23,6 & $(12,4-34,7)$ \\
Outros & 5 & 2,6 & $(14,5-37,4)$ \\
\hline
\end{tabular}

IC95\%: Intervalo de confiança 95\%. Nota: a variável "riscos da exposição solar" admitiu mais de uma resposta.

\section{Discussão}

\section{Resumo dos principais achados do estudo}

O fototipo dos ACS, avaliado na escala de Fitzpatrick, evidenciou a predominância dos tipos III e IV. Quando inquiridos acerca dos seus hábitos de exposição solar, observou-se que 48,2\% dos ACS se expunham ao sol durante 2 a 6 horas entre segunda e sexta-feira, todavia, a parte que se expõe por mais de 6 horas $(35,7 \%)$ também foi bastante expressiva.

Verificou-se que a maioria dos ACS utilizam filtros solares raramente ou nunca, 37,5\% e 21,4\% respectivamente, e que os filtros solares mais procurados foram aqueles com mais de $30 \mathrm{FPS}(64,4 \%)$. Além disso, a quantidade de ACS que escolhiam protetores solares por autoindicação $(35,7 \%)$ ou o faziam sob recomendação médica $(37,5 \%)$ foram equivalentes, o que poderia indicar falhas no conhecimento sobre a fotoproteção adequada, fato ratificado, diante da maior preferência por FPS maiores que 30 .

Ademais, a frequência de aplicação do filtro solar foi considerada inadequada, uma vez que 37,5\% e 16,1\%, aplicava somente uma vez no dia ou então quando iam à praia ou piscina, respectivamente, indicando que existe uma prática do cuidado com a exposição solar, porém restrita ao lazer e que não consegue se inserir na prática diária do trabalho e que quando está inserida ainda assim é insuficiente para uma boa proteção.

Quanto ao conhecimento acerca dos riscos e das consequências negativas da exposição solar, $100 \%$ dos ACS relataram conhecer algum, sendo os mais citados: o câncer de pele $(26,7 \%)$, as queimaduras solares $(21 \%)$, as manchas $(26,1 \%)$ e o envelhecimento da pele $(23,6 \%)$.

\section{Comparação com a literatura já existente}

Os ACS têm uma exposição solar excessiva, com a maioria se expondo de 2 a 6 horas semanais e mais de um terço deles se expondo ao sol por mais de 6 horas por semana. Além disso, o horário de exposição ao sol mais prevalente (55,4\% dos ACS) foi compreendido entre 10 horas da manhã e 3 da tarde. É de especial relevância o horário de exposição ao sol mais prevalente observado, visto que constitui o 
período em que a radiação solar torna-se potencialmente mais deletéria. ${ }^{7}$ Dessa forma, pode-se inferir que a provável razão para o ocorrido esteja relacionada com a jornada de trabalho diária desses profissionais, que os obriga diretamente à exposição solar duradoura. Tal comportamento de exposição ao sol em horários inadequados, assemelha-se ao encontrado por Araújo et al., ${ }^{8}$ Cardoso et al. ${ }^{9}$ e Lima et al. ${ }^{10}$

Além disso, segundo o Consenso Brasileiro de Fotoproteção, ${ }^{7}$ o cerne da fotoproteção baseia-se na utilização de filtros solares com Fator de Proteção Solar (FPS) adequado para cada indivíduo. Assim, mesmo o fototipo predominante encontrado ter sido o fototipo III e fototipo IV, que estão sujeitos a queimaduras solares moderadas e brandas, respectivamente. Isto não os isenta do uso de filtro solar ou outras medidas fotoprotetivas. $^{7}$

Em contraste a isso, foi observado prevalência de $58,9 \%$ dos ACS que utilizam filtros solares raramente ou nunca, dados que poderiam ser explicados devido ao fornecimento irregular e em alguns casos, inexistente, de filtros solares pelos gestores municipais de saúde para os agentes, além dos preços frequentemente inacessíveis dos filtros solares com FPS superior a 30.

Tal achado foi bastante heterogêneo ao ser comparado com a literatura, visto que o fornecimento de filtro solar pelas prefeituras não é obrigatório. Assim, os estudos com achados similares foram os de Araújo et al..$^{8}$ Cardoso et al. ${ }^{9}$ e de Lima et al. ${ }^{10}$, e os estudos que contrastaram foram o de Costa et al. ${ }^{11}$ e o de Souza et al. ${ }^{12}$ É importante ressaltar que alguns estudos não levaram em conta outros fatores importantes no uso do protetor solar, como a frequência de aplicação semanal e de reaplicação diária, ,8,10-12 o que limitou tal comparação.

Além disso, dos poucos ACS que relataram utilizar filtro solar, a frequência de aplicação foi considerada inadequada, uma vez que a maioria aplicava somente uma vez no dia ou então quando iam à praia ou piscina $(53,6 \%)$ contrapondo a recomendação de reaplicar o filtro solar de 2 em 2 horas ou quando se molhar, ${ }^{7}$ o que ocorreu provavelmente devido à desinformação sobre a necessidade de reaplicação do produto. Quanto aos itens de proteção mecânica, os quais também estão a critério do gestor público o fornecimento aos ACS, são adjuvantes ao uso de filtros solares e os mais utilizados foram guarda-sóis e sombrinhas (45,9\%), que, apesar de ofertarem certa proteção solar, esta é insuficiente à necessária para a maioria das pessoas. ${ }^{7}$

Quanto ao conhecimento acerca dos riscos e das consequências negativas da exposição solar, $100 \%$ dos ACS relataram algum entendimento dos malefícios desde o câncer ao próprio envelhecimento da pele. Entretanto, a presença desse conhecimento entra em conflito com a baixa prevalência do uso diário de filtros solares, o que é ratificado nos estudos de Cardoso et al., ${ }^{9}$ de Lima et al. ${ }^{10}$ e de Costa et al., ${ }^{11}$ os quais apontam que trabalhadores externos usualmente conhecem a necessidade do uso regular dos filtros solares, mas raramente adotam esse hábito, sendo necessário reforçar mais a educação permanente para os ACS. ${ }^{12}$

\section{Fortalezas e limitações do estudo}

A principal fortaleza do estudo foi a confirmação da alta exposição solar dos ACS na sua jornada de trabalho, bem como do não uso medidas de fotoproteção pelos mesmos. 
Quanto às limitações do estudo, pode-se destacar a casuística pequena que abrangeu apenas dois bairros situados na região Central de Belém, a fim de garantir uma melhor homogeneidade da amostra, sendo necessário expandir o estudo para mais bairros a fim de comparar com outras realidades locais.

\section{Implicações para a pesquisa na área e/ou para a prática dos profissionais.}

Este trabalho tem como implicação o apontamento de caminhos para a realização de trabalhos futuros e ressalta a necessidade de se criar programas que visam à saúde ocupacional dos servidores da área da saúde, especialmente os ACS, que abrangeriam vários aspectos, dentre eles: o psicológico, o social e o médico, no qual se inclui a fotoproteção, destaque desse estudo.

Outra implicação do estudo é o fortalecimento do tema dos cuidados com a pele dos trabalhadores externos, no caso dos ACS. Tema este ainda muito carente de um olhar que envolva mais o ACS para alertá-los dos perigos da exposição solar.

\section{Conclusão}

Conclui-se com o presente estudo, que os Agentes Comunitários de Saúde apresentam uma rotina semanal com alta exposição solar. A fotoproteção dos ACS foi considerada inadequada, uma vez que poucos relataram utilizar filtros solares e os que relataram o seu uso o faziam de maneira inadequada. Quanto à fotoproteção mecânica, a maioria utiliza algum meio, o que provavelmente justifica-se pelo baixo custo dessa modalidade de fotoproteção se comparada ao uso diário de filtros solares, facilitando o seu acesso.

De maneira geral, o conhecimento sobre os riscos e as consequências da exposição solar foi considerado excelente, contrastando com as práticas adotadas pelos Agentes. Tal fato gera a hipótese de que o problema pode estar vinculado ao custo alto dos protetores solares e a própria falta de hábito de seu uso. Contudo, estudos posteriores são necessários para comprovar essa associação.

\section{Contribuição dos autores}

Concepção e/ou delineamento do estudo: CMSRM, ALT, FCA e MSP; Aquisição, análise ou interpretação dos dados: ALT, FCA, TRMS e MSP; Redação preliminar: CMSRM, ALT, FCA TRMS e MSP; Revisão crítica da versão preliminar: CMSRM e MSP.

Todos os autores aprovaram a versão final e concordaram com prestar contas sobre todos os aspectos do trabalho.

\section{Agradecimentos}

Aos Agentes Comunitários de Saúde pela participação na presente pesquisa, a Secretaria Municipal de Saúde de Belém e a Universidade do Estado do Pará pelo apoio institucional fornecido.

\section{Conflito de interesses}

Declaram não haver. 


\section{Referências}

1. Barros IC. A importância da estratégia de saúde da família: contexto histórico [Monografia]. Teófilo Otoni: Universidade Federal de Minas Gerais; 2014.

2. Brasil, Ministério da Saúde. Portaria № 2.488, de 21 de outubro de 2011. Aprova a Política Nacional de Atenção Básica, estabelecendo a revisão de diretrizes e normas para a organização da Atenção Básica, para a Estratégia Saúde da Família (ESF) e o Programa de Agentes Comunitários de Saúde (PACS). Brasília: Ministério da Saúde; 2011. [acesso 2017 Out 5]. Disponível em: http://bvsms.saude. gov.br/bvs/saudelegis/gm/2011/prt2488_21_10_2011.html

3. Fraga OS. Agente comunitário de saúde: elo entre a comunidade e a equipe da ESF? [Monografia]. Governador Valadares: Universidade Federal de Minas Gerais; 2011.

4. Brasil. Ministério da Saúde. Secretaria de Atenção à Saúde. Departamento de Atenção Básica. Política Nacional de Atenção Básica. Brasília: Ministério da Saúde; 2012.

5. Kumar V, Abbas AK, Aster JC. Robbins \& Cotran - Bases Patológicas das Doenças. 9ª ed. Rio de Janeiro: Elsevier; 2016.

6. Azulay RD, Azulay DR, Azulay-Abulafia L. Dermatologia. 6ª ed. Rio de Janeiro: Guanabara Koogan; 2013.

7. Schalka S, Steiner D, Ravelli FN, Steiner T, Terena AC, Marçon CR, et al. Brazilian consensus on photoprotection. An Bras Dermatol. 2014;89(6 Suppl 1):1-74. http://dx.doi.org/10.1590/abd1806-4841.20143971

8. Araújo FC, Sousa BRM, Leite GG, Freitas LC, Lemos ELC, Pires CAA. Avaliação dermatológica de agentes comunitários de saúde sujeitos à fotoexposição em região tropical do Brasil. Sci Med. 2016;26(4):ID23897.http://dx.doi.org/10.15448/1980-6108.2016.4.23897

9. Cardoso FAMES, Mesquita GV, Campelo V, Martins MDCCE, Almeida CAPL, Rabelo RS, et al. Prevalence of photoprotection and its associated factors in risk group for skin cancer in Teresina, Piauí. An Bras Dermatol. 2017;92(2):206-10. http://dx.doi.org/10.1590/ abd1806-4841.20174831

10. Lima AG, Silva AMM, Soares CEC, Souza RAX, Souza MCMR. Fotoexposição solar e fotoproteção de agentes de saúde em município de Minas Gerais. Rev Eletr Enferm [Internet]. 2010;12(3):478-82. [acesso 2018 Maio 26]. http://dx.doi.org/10.5216/ree.v12i3.6156

11. Costa CMC, Silva VR, Costa LM, Silva BG, Moreira RS. Promovendo a saúde do trabalhador: enfoque no agente comunitário de saúde. In: Anais do 12ํㅡㄹ Congresso Brasileiro de Medicina de Família e Comunidade; 2013 Maio 29 Jun 2; Belém, PA, Brasil. Rio de Janeiro: Sociedade Brasileira de Medicina de Família e Comunidade; 2013.

12. Souza MCMR, Horta TG, Melo ES, Rocha FDB. Câncer de pele: hábitos de exposição solar e alterações cutâneas entre agentes de saúde em um município de Minas Gerais. Rev Enferm Cent O Min. 2016;1(6):1945-56. http://dx.doi.org/10.19175/recom.v0i0.920 